\title{
Zum Zusammenhang von Geschlechterungleichheiten in Bildung, Beruf und Karriere:
}

\section{Ein Ausblick}

Regula Julia Leemann \& Christian Imdorf

Published as:

Leemann, Regula Juila \& Imdorf, Christian (2011). Zum Zusammenhang von Geschlechterungleichheiten in Bildung, Beruf und Karriere: Ein Ausblick. In: A. Hadjar (ed.), Geschlechtsspezifische Bildungsungleichheiten. Systematischer Überblick zur Frage der Bildungsungleichheit zwischen den Geschlechtern. Wiesbaden: VSVerlag für Sozialwissenschaften, 417-440.

DOI 10.1007/978-3-531-92779-4_17

Original source of publication: http://www.springerlink.com/content/u0m8634lq96500p1/

The final publication is available at www.springerlink.com

\section{Kontaktadresse}

Prof. Dr. Regula Julia Leemann

Pädagogische Hochschule

Institut Sekundarstufe I und II

Clarastrasse 57

4058 Basel

$\mathrm{T}+41616901916$ (Direkt)

T +41616901900 (Zentrale)

F +41616901990

regula.leemann@fhnw.ch 


\begin{abstract}
Ziel der folgenden Ausführungen im abschließenden Teil dieses Sammelbands zur Entwicklung und Genese von geschlechtsspezifischen Bildungsungleichheiten ist es, den Blick zu öffnen in Richtung Berufsleben. Wie sind die verbesserten Bildungsmöglichkeiten von Frauen zu interpretieren? Ist es in den letzten Jahrzehnten gelungen, eines der grundlegendsten gesellschaftlichen Ungleichheitsverhältnisse zu beseitigen? Oder beginnt sich dieses sogar zu verkehren in eine gesellschaftliche Benachteiligung der Männer? Wir gehen bei unseren Überlegungen von der These aus, dass ein Abbau von Benachteiligungen der Frauen im Bildungssystem für sich genommen noch wenig aussagekräftig ist, wenn wir uns mit der klassischen soziologischen Frage der Persistenz bzw. des Wandels von gesellschaftlichen Ungleichheiten befassen wollen. Erst wenn die ganze Verknüpfung von Bildung und gesellschaftlicher Ungleichheit in den Blick genommen wird und sich dabei zeigt, dass Frauen ihre Bildungsgewinne auch in entsprechende Chancen im Beschäftigungssystem umsetzen können, sind ihre verbesserten Bildungschancen ein Gewinn für die Individuen und ein Fortschritt für die Gesellschaft - und erst dann könnten mögliche Bildungsvorteile von Frauen, wie sie in den vorliegenden Aufsätzen z.T. diagnostiziert werden, gar als neue gesellschaftliche Benachteiligungen von Männern skandalisiert werden.
\end{abstract}




\section{Einleitung}

Ziel der folgenden Ausführungen im abschließenden Teil dieses Sammelbands zur Entwicklung und Genese von geschlechtsspezifischen Bildungsungleichheiten ist es, den Blick zu öffnen in Richtung Berufsleben. Wie sind die verbesserten Bildungsmöglichkeiten von Frauen zu interpretieren? Ist es in den letzten Jahrzehnten gelungen, eines der grundlegendsten gesellschaftlichen Ungleichheitsverhältnisse zu beseitigen? Oder beginnt sich dieses sogar zu verkehren in eine gesellschaftliche Benachteiligung der Männer? Wir gehen bei unseren Überlegungen von der These aus, dass ein Abbau von Benachteiligungen der Frauen im Bildungssystem für sich genommen noch wenig aussagekräftig ist, wenn wir uns mit der klassischen soziologischen Frage der Persistenz bzw. des Wandels von gesellschaftlichen Ungleichheiten befassen wollen. Erst wenn die ganze Verknüpfung von Bildung und gesellschaftlicher Ungleichheit in den Blick genommen wird und sich dabei zeigt, dass Frauen ihre Bildungsgewinne auch in entsprechende Chancen im Beschäftigungssystem umsetzen können, sind ihre verbesserten Bildungschancen ein Gewinn für die Individuen und ein Fortschritt für die Gesellschaft - und erst dann könnten mögliche Bildungsvorteile von Frauen, wie sie in den vorliegenden Aufsätzen z.T. diagnostiziert werden, gar als neue gesellschaftliche Benachteiligungen von Männern skandalisiert werden.

Was meinen wir damit? Die Bedeutung von Bildung hat sich im Laufe der letzten 150 Jahre von einem persönlichen Gut, einem Mittel der individuellen Konsumption, hin zu einem gesellschaftlichen Gut gewandelt, das als Element des gesellschaftlichen Reichtums, als Triebkraft individueller und kollektiver Entwicklung, und als Moment des gesellschaftlichen Reproduktionsprozesses begriffen wird (Krais 1983: 217). Zu dieser gemeinhin geteilten Überzeugung bezüglich des Wertes und der Wichtigkeit von Bildung im gesellschaftlichen Reproduktionsprozess gehört auch die Vorstellung, dass mittels Bildung und der im Bildungssystem institutionalisierten Meritokratie gesellschaftliche Ungleichheitsverhältnisse legitimiert sind (Becker und Hadjar 2009; Solga 2005).

Konzeptionell können dabei zwei Aspekte unterschieden werden, welche zusammengenommen das Verhältnis von gesellschaftlicher Ungleichheitsstruktur und Bildung verständlich machen (Graf und Lamprecht 1991). Einerseits werden im pyramidal angelegten Bildungssystem über die Zertifizierung von (legitimen) Wissensbeständen, über die Ausdifferenzierung, Hierarchisierung und Kanalisierung von Bildungswegen sowie die damit einhergehende Konstruktion von höherer Bildung als knappes Gut Bildungsungleichheiten hergestellt. Es stellt sich hinsichtlich der Legitimation von sozialer 
Ungleichheit die empirisch zu beantwortende Frage, in welcher Beziehung die Ungleichheiten in den Bildungswegen und Bildungsergebnissen (Bildungsstatus) mit den institutionell verfestigten Positionen in der Sozialstruktur (Herkunftsstatus, Geschlechtsstatus, Migrationsstatus) der sich im Bildungssystem befindlichen nachwachsenden Generation stehen, inwiefern also gesellschaftliche Ungleichheiten in ungleiche Bildungslaufbahnen überführt werden. In den in diesem Band versammelten Aufsätzen wird dieser erste Aspekt hinsichtlich Geschlechterungleichheiten in den Blick genommen, indem die Entwicklungen in den Bildungsergebnissen nach Geschlecht in ihrem historischen Wandel dargestellt und mögliche Erklärungen dafür ausgebreitet werden.

Andererseits sind in modernen Gesellschaften Fragen der Verteilung von sozialen Gütern und Lebenschancen, d.h. die Regelungen des Zugangs zu gesellschaftlichem Ansehen, Einkommen und Einfluss sowie zu den damit verknüpften Privilegien weitgehend an das Bildungssystem und die institutionalisierte Form von Tauschbeziehungen, den Markt, übertragen worden (Bornschier und Aebi 1992: 544). Bildungsabschlüsse (Zertifikate, Titel) sind eine Art "Geldmittel" oder "Kapital", welche zu bestimmten "Wechselkursen" in andere Formen von sozialen Gütern - insbesondere im Berufs- und Beschäftigungssystem umgewandelt werden können. Voraussetzung dafür ist eine relativ enge Entsprechung der Hierarchien im Bildungs- und im Beschäftigungssystem. Bildungszertifikate berechtigen ihre Besitzer, bestimmte soziale Positionen innerhalb der gesellschaftlichen Sozialstruktur einzunehmen.

Es stellt sich hier die ebenfalls empirisch zu beantwortende Frage, welchen gesellschaftlichen Wert bestimmten Bildungswegen und -titeln zugesprochen wird, und in welcher Beziehung diese mit weiteren Bildungsmöglichkeiten sowie beruflichen Wegen stehen, inwiefern also die Umwandlung von Bildungsstatus in gesellschaftlichen Status, Laufbahn- und Lebenschancen legitim verläuft oder durch soziale Ungleichheitsverhältnisse in den verschiedenen gesellschaftlichen Teilsystemen moderiert wird.

Die im Anschluss an die Bildungszugewinne von Frauen zu verfolgende Frage ist deshalb, ob Frauen diese im weiteren Lebensverlauf auch in entsprechende berufliche Positionen und Karrierechancen umsetzen können. Führen die von Frauen eingeschlagenen Bildungswege im Vergleich zu den von Männern beschrittenen zu gleichen Beschäftigungschancen? Haben sich parallel zu den vergrößerten Bildungschancen auch ihre beruflichen Möglichkeiten verbessert und sind sie im Erwerbsleben womöglich daran, die Männer zu überholen? Um diese Zusammenhänge untersuchen zu können, sind selbstredend umfassende und komplexe Forschungsdesigns notwendig, welche mittels Kohorten- und 
Panelanalysen den Wandel von Bildungschancen, Berufswegen und beruflichen Statusindikatoren sowie deren Beziehungen einfangen können, sowie internationale Vergleichsstudien, um den Dimensionen der nationalspezifisch unterschiedlichen Institutionalisierungen von Bildung und Beschäftigung gerecht zu werden.

In den folgenden Ausführungen, welche sich einer institutionentheoretischen Herangehensweise verpflichten, beschränken wir uns im Sinne eines Ausblickes auf einige ausgewählte Aspekte des geschlechtsspezifischen Verhältnisses von Bildungswegen, Beruf und Karriere. Sie verweisen darauf, dass die proklamierten Bildungserfolge von Frauen bei genauerer Betrachtung relativiert werden müssen, da die in den gesellschaftlichen Institutionen Bildung, Arbeitsmarkt und Familie eingelassenen Geschlechterordnungen die Bildungs- und Berufswege von Frauen und Männern nach wie vor ungleich strukturieren. Um die Komplexität der Thematik etwas zu reduzieren, greifen wir in erster Linie auf empirische Resultate aus der Schweiz zurück, d.h. beschränken uns auf die spezifischen kulturellen und strukturellen Ausprägungen eines ausgewählten nationalstaatlich verfassten Gesellschaftssystems, das jedoch in vielen Bereichen Ähnlichkeiten mit Deutschland aufweist. Im Kapitel 2 und 3 befassen wir uns mit dem Zustandekommen von horizontaler Geschlechtersegregation in Bildung und Beruf und den Folgen für die Beschäftigungschancen von Frauen und Männern. Kapitel 4 fragt danach, ob Frauen ihre gestiegene Bildungspartizipation in eine entsprechende verbesserte berufliche Integration umwandeln können. In Kapitel 5 wird anhand einer eigenen Studie zu wissenschaftlichen Laufbahnen von Doktorierten des Schweizer Hochschulsystems für eine lebenslauftheoretische Sicht plädiert, welche die geschlechtsspezifisch ungleiche institutionelle Einbindung in die Familie sowie die Konstellation von Paaren miteinbezieht. Abschließend wird in Kapitel 6 eine Einschätzung vorgenommen zur Frage, ob die Bildungserfolge von Frauen in den letzten Jahren Anlass zur Befürchtung geben, dass sich das gesellschaftliche Geschlechterverhältnis in nächster Zeit zu Ungunsten der Männer umkehren wird.

\section{Vergeschlechtlichte Bildungswege}

Um die Frage der geschlechtsspezifischen Verhältnisse von Bildung, Beruf und Karriere zu analysieren, werden im Folgenden die Bildungswege von Frauen und Männern und hierbei insbesondere das Zustandekommen horizontaler beruflicher Segregation durch vergeschlechtlichte Bildungswege analysiert. In den OECD-Ländern - mit Ausnahme der 
Schweiz und der Türkei - schließen Frauen heute häufiger eine Ausbildung auf Sekundarstufe II (ISCED 3) ab als Männer (OECD 2009: 45). Beim Zugang zur tertiären Ausbildung (ISCED 5) haben Frauen Männer ebenfalls überholt - auch in der Schweiz. Der Frauenanteil auf Tertiärstufe beträgt in allen Ländern mehr als die Hälfte, im Durchschnitt aller Länder 54 Prozent (OECD 2008: 53). Die damit verbundenen Bildungslaufbahnen sind je nach Modellierung der nationalen Bildungssysteme sehr unterschiedlich (OECD 2009: 295ff.). ${ }^{1}$ Auf Sekundarstufe II sind allgemeinbildende, berufsbildungsvorbereitende und berufsbildende Bildungsgänge zu unterscheiden. Die berufsbildenden Wege sind nochmals zu differenzieren in solche mit rein schulischer Ausrichtung und solche des dualen Systems, in solche mit direkten Anschlussmöglichkeiten an das tertiäre Bildungssystem dank Hochschulzugangsberechtigung bzw. Maturität (ISCED 3a) und solche, welche dies nicht erlauben (ISCED 3b). Auf Tertiärstufe sind neben den klassisch universitären (ISCED 5a) auch stärker auf Berufsfelder ausgerichtete Fachhochschulen inkl. Pädagogische Hochschulen (ISCED 5a) oder höhere Fachschulen (ISCED 5b) vorhanden, wobei die Fachhochschulen und höheren Fachschulen bislang keine direkten Anschlussmöglichkeiten zu einem Doktoratsstudium eröffnen.

\subsection{Geschlechterunterschiede in den Bildungswegen auf Sekundarstufe II}

Diese Wege im berufsbildenden und allgemeinbildenden Bildungssystem - in ihrer Gestalt historisch gewachsen - sind keineswegs geschlechterneutral (für eine Übersicht zur Schweiz für die Sekundarstufe II vgl. Imdorf 2005: 69ff). Die Geschlechter wurden - schon von Anbeginn der Institutionalisierung des öffentlichen Bildungssystems - spätestens nach der obligatorischen Volksschulzeit auf unterschiedliche Bildungswege gelenkt, welche die weiteren Bildungsmöglichkeiten sowie daran anschließende beruflichen Wege kanalisierten (Eidgenössische Frauenkommission 2010; Imdorf 2005: 144ff; für Deutschland Krüger 1995: 209ff.). Dies wird im Folgenden zuerst für den berufsbildenden, anschließend für den allgemeinbildenden Weg dargestellt.

Berufsbildender Weg

\footnotetext{
${ }^{1}$ Für die Schweiz siehe SKBF 2010 und http://www.edudoc.ch/static/web/bildungssystem/grafik_bildung_d.pdf, für Deutschland Cortina et al. 2008.
} 
In der Schweiz beginnen innerhalb von zwei Jahren nach der obligatorischen Schulzeit rund zwei Drittel der Schulabgängerinnen und Schulabgänger eine Berufsausbildung (Hupka 2003: 35), wobei insbesondere in der Deutschschweiz dies in Form einer Lehre im dualen System erfolgt. Gemäß BBT (2010: 11f.) absolvieren nur 17 Prozent aller Berufslernender eine berufliche Vollzeitschule (Deutschschweiz 13 Prozent, Westschweiz 28 Prozent, Tessin 24 Prozent $)^{2}$. In der Deutschschweiz absolvieren Mädchen dabei häufiger eine berufliche Vollzeitschule als Jungen (Meyer 2009: 31). Diese geschlechtsspezifische Segregationslinie zwischen dualem und vollzeitschulischem System scheint sich jedoch in den letzten Jahrzehnten verkleinert zu haben. 1980 besuchten noch 21 Prozent der Frauen im Vergleich zu 9 Prozent der Männer eine Vollzeitschule. 1998 waren es noch 17 Prozent der Frauen und 11 Prozent der Männer (Borkowsky 2000), was auf die Gründung von Informatikerschulen sowie die wachsende Beliebtheit der Handelsschulen zurückzuführen ist.

Wie Hupka et al. (in diesem Band) zeigen, wählen Knaben generell häufiger den Weg über die Berufsbildung als Mädchen. Zwei Jahre nach Schulabschluss befinden sich rund 55 Prozent der Mädchen, jedoch 75 Prozent der Knaben in einer Berufsausbildung. Hirschi (2009) weist nach, dass dies zum einen mit dem kleineren Ausbildungsplatzangebot frauentypischer Berufe im Verhältnis zu männertypischen Ausbildungsberufen zusammenhängt, zum anderen mit dem tendenziell höheren schulischen Anspruchsniveau der frauentypischen Ausbildungsberufe, was Mädchen auf dem anforderungstieferen Niveau der Sekundarstufe I bei der Ausbildungsplatzsuche benachteiligt (Imdorf 2005: 144).

Die Einrichtung der Berufslehre - aus historischen Gründen stark im gewerblichindustriellen Wirtschaftssektor verankert (Jenzer 1998; Gonon 1998) - ist demnach immer noch stärker auf die Knaben ausgerichtet, auch wenn - im Zusammenhang mit dem Übergang der Berufsbildung in den Bereichen Gesundheit, Soziales und Kunst in die Verantwortung des Bundes - in den letzten Jahren für die von den Mädchen präferierten Berufe im sozialen und pflegerischen Bereich Berufslehren mit einem eidgenössisch anerkannten Fähigkeitszeugnis (EFZ) geschaffen wurden (Fachangestellte Betreuung, Fachangestellte Gesundheit), welche nach der Lehre Anschlussmöglichkeiten an die entsprechenden Berufsausbildungen an den Fachhochschule erlauben (SKBF 2010: 164).

\footnotetext{
${ }^{2}$ Seibert et al. (2009: 599) berücksichtigen in ihren Berechnungen nur die Ausbildungsplätze des ersten Ausbildungsjahres und berechnen auf dieser Grundlage für das Jahr 2004 mit 19,6\% Vollzeitberufsschulen gegenüber 80,4\% dualen Ausbildungen einen etwas höheren Anteil an Vollzeitschulen an sämtlichen voll qualifizierenden Berufsausbildungen. Dieser variiert dabei beträchtlich nach Kanton und schwankt zwischen $58 \%$ (Kt. Genf) und 0\% (Kt. Nidwalden) (ebd., 601).
} 
Über die letzten Jahrzehnte sind die geschlechtsspezifischen Berufswahlmuster auf der Sekundarstufe II von großer Kontinuität gekennzeichnet. Wie Tabelle $1 \mathrm{zu}$ entnehmen ist, kommt es auf der Hitliste der zehn am häufigsten gewählten Berufe zwischen 1980 und 2000 kaum zu Veränderungen. Neben den von beiden Geschlechtern bevorzugten Berufsgruppen kaufmännische Angestellte und Büroberufe sowie Verkäufer und Detailhandelsangestellte figurieren Elektromonteure und -installateure, Mechaniker, Automechaniker, Maurer und Schreiner, Hoch- und Tiefbauzeichner, Landwirte und Küchenpersonal bei den Knaben an wichtiger Stelle. Primarlehrerin, medizinische Praxisassistentin und Arztgehilfin, Apothekenhelferin, Krankenschwester und Coiffeuse, Kindergärtnerinnen und Zahnarztgehilfinnen sind durchwegs prioritär für die Mädchen. 
Tabelle 1: Die zehn häufigsten Ausbildungsberufe, 22-24jährige Frauen und Männer mit Ausbildungsabschluss Sekundarstufe II, 1980-2000

\begin{tabular}{|c|c|c|c|}
\hline Rang & $\begin{array}{c}\text { Männer Sekundarstufe II } \\
1980 \\
(22-24 \text { Jährige }) \\
\end{array}$ & $\begin{array}{c}\text { Männer Sekundarstufe II } \\
1990 \\
(22-24 \text { Jährige }) \\
\end{array}$ & $\begin{array}{c}\text { Männer Sekundarstufe II } \\
2000 \\
(22-24 \text { Jährige }) \\
\end{array}$ \\
\hline 1 & $\begin{array}{c}\text { Kaufmännische Angestellte, } \\
\text { Büroberufe }\end{array}$ & $\begin{array}{c}\text { Kaufmännische Angestellte, } \\
\text { Büroberufe }\end{array}$ & $\begin{array}{c}\text { Kaufmännische Angestellte, } \\
\text { Büroberufe }\end{array}$ \\
\hline 2 & $\begin{array}{c}\text { Elektromonteure und - } \\
\text { installateure }\end{array}$ & Mechaniker & Elektromonteure und-installateure \\
\hline 3 & Mechaniker & Elektromonteure und-installateure & Automechaniker \\
\hline 4 & Automechaniker & Automechaniker & Mechaniker \\
\hline 5 & Maurer & Landwirte & Hoch- und Tiefbauzeichner \\
\hline 6 & Landwirte & Schreiner & $\begin{array}{c}\text { Verkäufer, } \\
\text { Detailhandelsangestellte }\end{array}$ \\
\hline 7 & Hoch- und Tiefbauzeichner & Maurer & Küchenpersonal \\
\hline 8 & Schreiner & Küchenpersonal & Schreiner \\
\hline 9 & $\begin{array}{c}\text { Verkäufer, } \\
\text { Detailhandelsangestellte }\end{array}$ & Verkäufer, Detailhandelsangestellte & Elektronikerberufe \\
\hline 10 & $\begin{array}{l}\text { Primarlehrer } \\
\text { Anteil der } 10 \text { häufigsten } \\
\text { Ausbildungsberufe an allen } \\
\text { Ausbildungsberufen: } 52 \%\end{array}$ & $\begin{array}{c}\text { Maler, Tapezierer } \\
\text { Anteil der } 10 \text { häufigsten } \\
\text { Ausbildungsberufe an allen } \\
\text { Ausbildungsberufen: } 48 \%\end{array}$ & $\begin{array}{c}\text { Maurer } \\
\text { Anteil der } 10 \text { häufigsten } \\
\text { Ausbildungsberufe an allen } \\
\text { Ausbildungsberufen: } 46 \%\end{array}$ \\
\hline
\end{tabular}

\begin{tabular}{|c|c|c|c|}
\hline Rang & $\begin{array}{c}\text { Frauen Sekundarstufe II } \\
1980 \\
(22-24 \text { Jährige })\end{array}$ & $\begin{array}{c}\text { Frauen Sekundarstufe II } \\
1990 \\
(22-24 \text { Jährige })\end{array}$ & $\begin{array}{c}\text { Frauen Sekundarstufe II } \\
2000 \\
(22-24 \text { Jährige })\end{array}$ \\
\hline 1 & $\begin{array}{c}\text { Kaufmännische Angestellte, } \\
\text { Büroberufe }\end{array}$ & $\begin{array}{c}\text { Kaufmännische Angestellte, } \\
\text { Büroberufe }\end{array}$ & $\begin{array}{c}\text { Kaufmännische Angestellte, } \\
\text { Büroberufe }\end{array}$ \\
\hline 2 & $\begin{array}{c}\text { Verkäuferinnen, } \\
\text { Detailhandelsangestellte }\end{array}$ & $\begin{array}{c}\text { Verkäuferinnen, } \\
\text { Detailhandelsangestellte }\end{array}$ & $\begin{array}{c}\text { Verkäuferinnen, } \\
\text { Detailhandelsangestellte }\end{array}$ \\
\hline 3 & Primarlehrerinnen & Coiffeusen & Primarlehrerinnen \\
\hline 4 & $\begin{array}{c}\text { Medizinische Praxisassistent., } \\
\text { Arztgehilfinnen }\end{array}$ & Krankenschwestern & Coiffeusen \\
\hline 5 & Krankenschwestern & $\begin{array}{c}\text { Medizinische Praxisassistent., } \\
\text { Arztgehilfinnen }\end{array}$ & Krankenschwestern \\
\hline 6 & Coiffeusen & Primarlehrerinnen & $\begin{array}{c}\text { Medizinische Praxisassistent., } \\
\text { Arztgehilfinnen }\end{array}$ \\
\hline 7 & $\begin{array}{l}\text { Spitalgehilfinnen, } \\
\text { Hilfsschwestern }\end{array}$ & Zahnarztgehilfinnen & Apothekenhelferinnen \\
\hline 8 & Kindergärtnerinnen uvB & Andere Berufe des Gastgewerbes & Zahnarztgehilfinnen \\
\hline 9 & $\begin{array}{l}\text { Teleoperatricen und } \\
\text { Telefonistinnen }\end{array}$ & Apothekenhelferinnen & Kindergärtnerinnen uvB \\
\hline 10 & $\begin{array}{c}\text { Schneiderinnen } \\
\text { Anteil der } 10 \text { häufigsten } \\
\text { Ausbildungsberufe an allen } \\
\text { Ausbildungsberufen: } 77 \%\end{array}$ & $\begin{array}{c}\text { Servicepersonal } \\
\text { Anteil der } 10 \text { häufigsten } \\
\text { Ausbildungsberufe an allen } \\
\text { Ausbildungsberufen: } 66 \%\end{array}$ & $\begin{array}{c}\text { Floristinnen } \\
\text { Anteil der } 10 \text { häufigsten } \\
\text { Ausbildungsberufe an allen } \\
\text { Ausbildungsberufen: } 60 \%\end{array}$ \\
\hline
\end{tabular}

Datenquelle: Eidgenössische Volkszählungen, BFS

Darstellung aus Leemann und Keck 2005: 75

Kursiv: In allen drei Kohorten gehört dieser Beruf zu den zehn häufigsten erlernten Ausbildungsberufen.

Kursiv und fett: In allen drei Kohorten und sowohl für Frauen wie für Männer gehört dieser Beruf zu den zehn häufigsten erlernten

Ausbildungsberufen. "uvB" (und verwandte berufe)

Die Wahl von gegengeschlechtlichen Berufen ist nach wie vor mit großen Hürden verbunden. Im Kanton Zürich beispielsweise mündete im Jahr 2004 nur eine kleine Minderheit von 5 Prozent (9,1 Prozent der Frauen vs. 1,7 Prozent der Männer) in einen gegengeschlechtlichen Beruf. 61 Prozent der Frauen gegenüber 33 Prozent der Männer befanden sich in einem der gemischtgeschlechtlichten Berufe, welche tendenziell höhere schulische Anforderungen verlangen (Abraham und Arpagaus 2008: 213ff.). Die Berufswahl ist - so können wir 
festhalten - nach wie vor von ausgeprägten horizontalen geschlechtsspezifischen Segregationen gekennzeichnet, welche trotz bemerkenswerten kulturellen Wandlungsprozessen ein außerordentliches Beharrungsvermögen erkennen lassen (SKBF 2010: 158).

Auszubildende im dualen System der Schweiz haben seit 1970 die Möglichkeit, statt der die Lehre begleitenden Berufsfachschule die anspruchsvollere Berufsmittelschule zu besuchen (Criblez 2001). 1994 wurde sie in die sog. Berufsmaturitätsschule BMS überführt, welche erlaubt, eine Berufsmaturität zu erlangen und daran anschließend an eine Fachhochschule überzutreten. ${ }^{3}$ Im Jahr 2009 wurden 56 Prozent der Berufsmaturitätszeugnisse an Männer ausgestellt, 44 Prozent an Frauen (BFS 2010: 45, eigene Berechnungen). Damit ist der Anteil der Frauen, der diesen Schultyp besucht, von 30 Prozent im Jahre 1988 (Gonon 2001: 63) auf heute 44 Prozent zwar beträchtlich gewachsen. Die Verteilung der Berufsmaturitätstitel, die nach beruflichen Richtungen ausgestellt werden, sind aufgrund der geschlechtsspezifischen Berufwahlmuster jedoch ebenfalls hochgradig geschlechtersegregiert - gesundheitliche, soziale sowie gestalterische Titel gehen mehrheitlich an Frauen, naturwissenschaftliche und technische Titel an Männer. Ausgeglichen ist das Geschlechterverhältnis bei den kaufmännischen und gewerblichen Titeln (vgl. BFS 2010). Diese Geschlechtertypik hat in der Folge Auswirkungen auf die Geschlechterverteilung an den verschiedenen Fachhochschulen, da die Berufsmaturitätstypen auf den Fachhochschulstudiengang abgestimmt sind und damit den weiteren Bildungs- und Berufsweg vorspuren (SKBF 2010: 147). Frauen treten des Weiteren nach einer Berufsmaturität mit einer geringeren Wahrscheinlichkeit in ein Fachhochschulstudium über als Männer, wobei sich die Geschlechterunterschiede in den letzten Jahren stetig abgebaut haben (SKBF 2010: 156f.).

\section{Allgemeinbildender Weg}

Insgesamt befinden sich heute rund 20 Prozent der Knaben und 33 Prozent der Mädchen zwei Jahre nach der obligatorischen Schule in allgemeinbildenden Schulen (Gymnasien,

\footnotetext{
${ }^{3}$ Die Berufsmaturitätsschule wird während der Lehrzeit besucht und umfasst rund 1 Tag mehr Unterricht pro Woche als die Berufsfachschule (D: Berufsschule). Sie kann aber auch nach Abschluss der Berufslehre absolviert werden, dauert ein Jahr (Vollzeitschule) oder bis zu zwei Jahren (Teilzeitschule) und wird ebenfalls mit der Berufsmaturität abgeschlossen.
} 
Fachmittelschulen) der Sekundarstufe II (Hupka et al. in diesem Buch), Mädchen sind demnach klar übervertreten. Im Kanton Zürich hatten die weiblichen Jugendlichen schon um 1960 mit einer Mittelschülerquote von 10.5 Prozent mit den männlichen Jugendlichen (10.7 Prozent) gleichgezogen. Wenn jedoch die Schultypen betrachtet werden, wird erkennbar, dass die Mädchen damals in den sog. Diplommittelschulen DMS übervertreten waren, die Gymnasien dagegen noch von den Knaben dominiert wurden. Erst 1980 näherte sich die Gymnasialquote der Mädchen jener der Knaben an (Rieger 2001: 46).

Die Wurzeln dieser geschlechtsspezifischen Wege gehen bis ins 19. Jahrhundert zurück. Als die Mädchen um die Jahrhundertwende immer stärker in die höheren Bildungsgänge strömten, wurden sie in die auf die bürgerliche Frauenrolle und auf Frauenberufe vorbereitenden höheren Töchterschulen und Handelsdiplomschulen - die Vorläufer der DMS - gelenkt (Rieger 2001), was dazu führte, dass diese Bildungsgänge bis heute mehrheitlich von Mädchen besucht werden (Imdorf 2005: 146). Im Jahre 2007/08 waren rund drei Viertel der Schülerschaft Mädchen (SKBF 2010: 164).

In den letzten Jahren sind die DMS in sog. Fachmittelschulen FMS überführt worden, welche mit einer Fachmaturität abgeschlossen werden können. Diese bieten jedoch nach vier Jahren keinen berufsqualifizierenden Abschluss auf Sekundarstufe II, sondern bereiten auf einen Studiengang im nicht-universitären Bereich vor (SKBF 2010: 166). Die FMS ist ein institutionalisierter Weg zu Ausbildungen in den klassischen Frauenberufen im pflegerischen, sozialen sowie pädagogischen Bereich ${ }^{4}$, welche seit rund zehn Jahren an Fachhochschulen ${ }^{5}$ angesiedelt sind. Bis heute gilt deshalb das Fazit von Krüger (1995: 211), dass dieser frauenspezifische Weg im Vergleich zum Weg über die duale Lehre eher höhere Ausbildungsvoraussetzungen erfordert ${ }^{6}$ (und damit die schulischen Vorsprünge von Mädchen sozusagen absorbiert werden), erheblich längere Ausbildungszeiten benötigt sowie finanziell größere Aufwendungen mit sich bringt, da die Lernenden in einer Berufslehre ein Lehrgeld erhalten und nach drei bis vier Jahren in den Arbeitsmarkt übertreten können. Für unsere Thematik ist des Weiteren die Tatsache interessant, dass die Fachmaturität nach vier Jahren nachobligatorischer Ausbildungszeit keinen direkten Zugang zu den universitären

\footnotetext{
${ }^{4}$ Für die Ausbildung zur Lehrerin/zum Lehrer auf Volksschulstufe (nur gewisse Kantone) und die Ausbildung für den Unterricht auf Sekundarstufe I wird jedoch eine gymnasiale Maturität verlangt. Mit der Erweiterung der Profile im Zusammenhang mit der Umwandlung von DMS in die FMS werden weitere Berufsfelder wie Bibliothekswesen, Tourismus oder Musik und Theater anvisiert.

${ }^{5}$ Im Gegensatz zur französischsprachigen Schweiz verbleiben in der deutschsprachigen Schweiz jedoch einige Ausbildungen auf der Stufe der höheren Fachschulen (SKBF 2010: 164).

${ }^{6}$ Die meisten Schüler/innen kommen aus der Sekundarstufe I mit erweiterten Ansprüchen, oder wechseln aus anderen allgemeinbildenden Schulen der Sekundarschule II. Nur 3\% haben die Sekundarstufe mit Grundansprüchen besucht (SKBF 2010: 164).
} 
Hochschulen ermöglicht ${ }^{7}$, im Gegensatz zum Weg über die Berufsbildung mit Berufsmaturität, bei dem neben dem direkten Übergang auf eine Fachhochschule eine sogenannte Passerelle (Übergang) zu den Universitäten eingerichtet ist. Mit einer Ergänzungsprüfung kann ein universitäres Hochschulstudium begonnen werden.

Ab den 1990er Jahren begannen die Mädchen die Knaben bezüglich Gymnasialbesuchs zu überholen, was u.a. auch auf die Auflösung der seminaristischen Ausbildung zur Lehrerin/zum Lehrer der Volksschule zurückgeführt werden kann (SKBF 2010: 122). Der aktuelle Anteil der Mädchen im Gymnasium von 57 Prozent wird gemäß Prognosen bis Ende dieser Dekade konstant bleiben (Babel und Strubi 2010). Zurzeit liegt die Maturitätsquote bei den Frauen bei 22.8 Prozent (Vergleichsgruppe sind alle 19-Jährigen), bei den Männern bei 15.8 Prozent (SKBF 2010: 136).

Mädchen und Knaben wählen im Gymnasium unterschiedliche Schwerpunktfächer (fachliche Profile). Die Mädchen sind im neusprachlichen, musischen sowie im Profil PPP (Philosophie, Pädagogik, Psychologie) übervertreten. Von diesen Profilen aus wird seltener ein universitäres Studium und öfters ein Studium an einer pädagogischen Hochschule oder Fachhochschule angestrebt (Ramseier et al. 2005: 151-155). Größere geschlechtsspezifische Ungleichverteilungen zugunsten der Knaben finden sich im Profil PAM (Physik \& angewandte Mathematik) und im Profil Wirtschaft \& Recht. Von diesen Profilen aus wird sehr oft ein Universitätsstudium angestrebt. Im Ergebnis planen Mädchen am Ende des Gymnasiums signifikant seltener, an eine universitäre Hochschule überzutreten als Knaben, beabsichtigen dagegen häufiger, eine berufliche Ausbildung an einer Pädagogischen Hochschule oder Fachhochschule zu beginnen (Denzler und Wolter 2008). Das gewählte Profil hat nicht nur Auswirkungen auf den Hochschultyp, der nach der Maturität angepeilt wird, sondern auch auf die Studienwahl an der Universität (Bieri Buschor et al. 2008), vor allem in den Bereichen Sprachen und Mathematik.

Als Fazit können wir festhalten: Die mit den erreichten Bildungstiteln sich eröffnenden weiteren Bildungswege auf der Sekundarstufe II sind nicht gleichwertig. Zu erwähnen ist insbesondere, dass bei dem von Frauen bevorzugten Weg über die Fachmittelschulen keine Passerelle an die Universitäten eingerichtet ist, im Gegensatz zum Weg über die Berufsmaturität. Die von Mädchen und Knaben eingeschlagenen Bildungswege stellen im

\footnotetext{
${ }^{7}$ Die Abgänger einer Fachmaturität können nur über den Weg eines erworbenen Bachelorabschlusses an einer Fachhochschule anschließend in ausgewählte Studiengänge (z.T. auch auf Masterniveau) an der Universität übertreten. In diesem Bereich ist zurzeit im Schweizer Bildungssystem sehr viel in Bewegung, so dass es nicht möglich ist, generalisierende Aussagen über Zugangswege zu machen.
} 
Weiteren unterschiedliche schulische Anforderungen. Die von Mädchen präferierten Berufslehren sowie die rein schulischen, von Mädchen häufiger besuchten Wege über die Gymnasien und Fachmittelschulen sind intellektuell und kognitiv anspruchsvoller. Mädchen müssen also mehr leisten, um zum gleichen Bildungstitel zu kommen. Sie bringen aber dadurch bessere Voraussetzungen für die Aneignung der zukünftig immer wichtiger werdenden allgemeinen, überfachlichen "Schlüsselkompetenzen" mit (Kupfer 2010).

Berufsqualifizierende Abschlüsse, bei denen die jungen Männer überrepräsentiert sind, erlauben einen sofortigen, relativ gut abgesicherten Übergang ins Beschäftigungssystem und damit einen frühen Aufbau einer beruflichen Laufbahn mit entsprechenden Einkommensmöglichkeiten. Allgemeinbildende Abschlüsse dagegen - von Mädchen präferiert - erfordern weitere zeitliche und finanzielle Investitionen in Bildung, um im zertifikationsorientierten Beschäftigungssystem längerfristig gute berufliche Chancen zu erhalten. Der Strukturwandel Richtung Dienstleistungsgesellschaft wird innerhalb des Ausbildungssystems in erster Linie von den Frauen vollzogen. Das Beharrungsvermögen der männlichen Berufswahl insbesondere auf Sekundarstufe II kann inzwischen für die jungen Männer auch ein Beschäftigungsrisiko darstellen und muss von ihnen beim Übergang ins Berufsleben individuell durch einen Berufswechsel, verbunden mit einem sektoralen Wechsel, bewältigt werden (Leemann und Keck 2005).

\subsection{Geschlechterunterschiede in den Bildungswegen auf Tertiärstufe}

Die im vorhergehenden Kapitel geschilderten geschlechtsspezifischen Differenzen in den eingeschlagenen Bildungswegen auf Sekundarstufe II ziehen eine unterschiedliche Verteilung der Geschlechter auf die einzelnen Studienfächer auf der Tertiärstufe (Fachhochschulen und Universitäten) nach sich. Im internationalen Vergleich fällt vor allem der tiefe Anteil der Frauen in den Natur- und Technischen Wissenschaften auf (OECD 2008: 53). Diese Unterrepräsentanz der Frauen in den sog. MINT-Fächern (Mathematik, Informatik, Naturwissenschaft und Technik) auf Tertiärniveau ist sehr ausgeprägt: Im Vergleich mit 44 verschiedenen Ländern nimmt die Schweiz einen Spitzenplatz ein (Charles und Bradley 2009: 942). Dieses "sex segregation regime" wird von Charles und Bradley (2009) mit den in (post)modernen Gesellschaften ausgeprägten Geschlechterideologien bezüglich Fähigkeiten von Frauen und Männern in Zusammenhang gebracht. Es ist jedoch nicht nur kulturell institutionalisiert, sondern auch durch die bildungsbiografisch frühe Berufs- und Profilwahl bedingt. 
Der Frauenanteil in den Fachhochschulen hat in den letzten Jahren zugenommen, und seit 2006 bilden Frauen erstmals die Mehrheit der Eintretenden. ${ }^{8}$ Dies ist vor allem auf strukturelle Veränderungen in der Fachhochschullandschaft zurückzuführen. Im Zuge der Tertiarisierung der Lehrpersonenausbildung in den letzten Jahren wurden die Pädagogischen Hochschulen, und damit viele Frauen, in die Fachhochschullandschaft integriert. Die Geschlechter verteilen sich - bedingt durch die geschilderten kanalisierenden und geschlechtlich geprägten Bildungswege - z.T. sehr ungleich auf die einzelnen Fachhochschulen. So sind im Jahre 2010 im Bereich Gesundheit nur knapp 14 Prozent, im Bereich angewandte Linguistik (Übersetzung und Dolmetschen) 18 Prozent männliche Studierende zu finden. Im Bereich Technik und IT gibt es hingegen nur 8 Prozent weibliche Studierende, im Bereich Architektur, Bau- und Planungswesen 26 Prozent. Zu den geschlechterausgeglichenen Studiengängen können Musik, Theater und andere Künste (Frauenanteil 55 Prozent) oder Wirtschaft und Dienstleistungen (Frauenanteil 45 Prozent) gezählt werden.

Auch an den Universitäten treten mit 52 Prozent mehr Frauen als Männer ein. Die Frauen haben im Studienjahr 2001/02 die Männer anzahlmäßig das erste Mal überholt (Kunz 2010: 28ff). Die geschlechtsspezifisch unterschiedliche Fächerwahl ist sehr ausgeprägt (Franzen et al. 2004), mit über die letzten Jahrzehnte stabilen Präferenzen der Frauen für Geistes-, Sozial- und Sprachwissenschaften, und denjenigen der Männer für Ingenieurwissenschaften (Leemann und Keck 2005: 77). Medizin, die Rechts- und die Wirtschaftwissenschaften dagegen werden von beiden Geschlechtern in ähnlichen Ausmaßen gewählt. Im Ergebnis sind die Studienfächer durch unterschiedliche Geschlechteranteile gekennzeichnet (SKBF 2010: 202). Dennoch sind auf Tertiärstufe eher Wandlungsprozesse erkennbar und die Geschlechterordnungen scheinen hier im Vergleich zur Sekundarstufe II weniger rigide zu sein (Leemann und Keck 2005: 122; Buchmann und Kriesi 2009).

Die geschlechtsspezifische Struktur in der Studienwahl hat Implikationen für die letzte Bildungsstufe, das Doktorat. In den Sozial- und Geisteswissenschaften, welche sich durch eine hohe Konzentration von Frauen auszeichnen, sind die Geschlechterunterschiede in den Promotionschancen zu Ungunsten der Frauen am größten, und auch bei den jüngsten Jahrgängen mit Hochschulabschluss 1999-2001 haben innerhalb von fünf Jahren doppelt so viele Männer wie Frauen einen Doktortitel erworben (Dubach 2010: 48f). Mit Blick auf die für wissenschaftliche Laufbahnen in einigen Disziplinen relevante Habilitation kann ergänzt

\footnotetext{
${ }^{8}$ Siehe http://www.bfs.admin.ch/bfs/portal/de/index/themen/15/06/key/ind6.indicator.60202.602.html (Zugriff: 7.9.2010).
} 
werden, dass auch hier in den Sozial- und Geisteswissenschaften innerhalb von 12 Jahren nach Erhalt des Doktorats Frauen signifikant seltener habilitieren (Dubach 2010: 54).

Wie wir gezeigt haben, ist die geschlechtsspezifische Segregation im Bereich der Berufs- und Hochschulbildung nach wie vor markant und zeigt starke Beharrungstendenzen. In den letzten Jahrzehnten veränderten sich durch den Zustrom der Frauen die Geschlechterordnungen nur in einigen Fächern im Hochschulbereich. In der Schweiz gibt es demnach wenig Evidenz für ein säkulares "degendering" in Bildungsinstitutionen (Charles und Bradley 2009: 959).

\section{Vergeschlechtlichte Wege vom Bildungs- ins Beschäftigungssystem}

Das stark ausdifferenzierte Schweizer Ausbildungssystem strukturiert durch seine berufliche Ausrichtung sowohl der traditionellen Berufsbildung als auch der tertiären Studiengänge die horizontale Geschlechtersegregation auf dem Arbeitsmarkt und die damit verbundenen beruflichen und sozialen Ungleichheiten zwischen Männern und Frauen maßgeblich vor (Charles 2005; Charles und Buchmann 1994, Buchmann und Kriesi 2009; Smith und Steinmetz 2008). ${ }^{9}$ Auf der Ebene der konkreten Berufswahlen und des ersten ausgeübten Berufes zeigen Analysen der Volkszählungen zwischen 1970 und 2000, dass eine frappant hohe Kongruenz zwischen der Struktur der Berufsausbildungen und derjenigen der ausgeübten Tätigkeiten vorhanden ist. Ein Grossteil der Erwerbstätigen findet sich im selben Beruf oder zumindest in einem berufsnahen Tätigkeitsfeld wieder (Leemann und Keck 2005).

Verantwortlich dafür sind zum einen die ausgeprägte berufsfachliche Segmentierung des Schweizer Arbeitsmarkts und die damit einhergehenden beruflichen Mobilitätsbarrieren (Sacchi, in Vorbereitung). Der über die Berufswahl eingeschlagene berufliche Weg ist nur schwer zu revidieren (Charles und Buchmann 1994). Zum anderen zwingt das Schweizer wie auch das Deutsche Bildungssystem Jugendliche bereits in jenem Alter, in welchem die geschlechtstypischen Identitätsbildungsprozesse ausgeprägt sind, sich beruflich zu orientieren. Geschlechtertypische berufliche Orientierungen sind unter dieser Voraussetzung besonders verbreitet, ohne dass sich die weiblichen Jugendlichen zu diesem Zeitpunkt bereits die praktischen Nachteile von Frauenberufen bewusst sind (Buchmann und Charles 1995: 73; Abraham und Arpagaus 2008: 223). Geht man von der begründeten Annahme aus, dass Jugendliche die Entscheidungen zu ihrer beruflichen Zukunft schrittweise fällen, dann erklärt

\footnotetext{
${ }^{9}$ Für Deutschland siehe die wegweisende Studie Ende der 1980er Jahre von Blossfeld (1989).
} 
die Ungleichheit bei der Wahl der Ausbildung rund 92 Prozent der geschlechtsspezifischen Gesamtsegregation auf dem Arbeitsmarkt (Flückiger und Falter 2004: 41).

Das Berufsprinzip ist in Westdeutschland (im Gegensatz zur ehemaligen DDR) kein geschlechtsneutraler Mechanismus der Arbeitsmarktallokation (Solga und Konietzka 2000). Die verminderte gesellschaftliche Wertschätzung von frauentypischen Tätigkeiten (gemessen an Entlöhnung, Prestige, Aufstiegschancen) resultiert in einer geschlechtsspezifisch differenten Wirksamkeit der Verwertbarkeit von Berufsabschlüssen. Dies gilt auch für die Schweiz, wo die vertikale Geschlechtersegregation des Arbeitsmarktes im internationalen Vergleich besonders stark mit der horizontalen Segregation zusammenhängt (Bridges 2003). So zeigt Jann (2008) anhand der Schweizerischen Arbeitskräfteerhebung in der Schweiz für den Zeitraum 1991-2006, dass die horizontale berufliche Segregation nach Geschlecht (Frauenanteil in Beruf und Branche) ein wichtiger Faktor für Erklärung von Einkommensungleichheiten zwischen den Geschlechtern ist. Zum selben Ergebnis kommen Holst und Busch (2009), welche den "gender pay gap" für Führungspositionen in der Privatwirtschaft (Deutschland) untersuchen. Für Deutschland konnte Trappe (2006: 66ff) zeigen, dass Frauen in Westdeutschland einem deutlich höheren Risiko ausgesetzt sind, unterhalb ihrer Qualifikation zu arbeiten, wenn sie einen frauentypischen Beruf (im Unterschied zu einem Misch- oder Männerberuf) erlernt haben. Dieses Risiko ist für Männer dann gegeben, wenn sie nicht einen männertypischen Beruf erlernt haben.

Mütter mit einem typischen „Frauenberuf“ sind im Weiteren seltener erwerbstätig als Mütter in einem Männerberuf oder in einem geschlechtergemischten Beruf (vgl. Buchmann et al. 2002). Männerberufe oder integrierten Berufe bieten dank größerer Flexibilität in der Arbeitsgestaltung die besseren Möglichkeiten, Beruf und Familie zu vereinbaren. Diese Berufe fördern zudem eine kontinuierliche Erwerbstätigkeit, weil ein längerer Unterbruch zu erhöhten Opportunitätskosten führt. Die typischen Frauenberufe in der Kategorie wie beispielsweise Detailhandelsangestellte, Friseurin oder Betagtenbetreuerin sind dagegen infolge ungünstiger Arbeitsbedingungen wie Schichtarbeit, Wochenendarbeit und langen Arbeitszeiten nur schwer mit den Zeiten der öffentlichen Kinderbetreuungsstätten und Schulen zu vereinbaren (Jurczyk 1993).

\section{Geschlechterungleichheiten bei der Umwandlung von Bildungstiteln in berufliche Positionen und Laufbahnchancen}


Wie gezeigt wurde, haben Frauen in der Schweiz bezüglich formaler Bildung aufgeholt, die damit verbundenen schulischen und beruflichen Bildungswege unterscheiden sich jedoch zwischen den Geschlechtern nach wie vor in ihrer fach- und berufsspezifischen Ausrichtung. Im Folgenden gehen wir der Frage nach, ob Frauen ihre gestiegene Bildungspartizipation in eine entsprechende verbesserte berufliche Integration umwandeln können. Wie Buchmann et al. (2002) sowie Hecken (2006) in der Aufarbeitung des Forschungsstandes für die Schweiz und Deutschland zeigen, führt das gestiegene Bildungsniveau zu einer stärkeren Berufsorientierung von Frauen, d.h. zu weniger und kürzeren Erwerbsunterbrechungen im Zusammenhang mit der Gründung einer Familie. Seit 1970 hat die Erwerbsbeteiligung von Frauen als auch der Anteil an Erwerbstätigen markant zugenommen. Gleichzeitig arbeiten immer mehr Frauen Teilzeit (Buchmann und Kriesi 2008). Frauen und Männer unterscheiden sich heute kaum in ihrem durchschnittlichen Umfang der Erwerbstätigkeit, solange sie kinderlos sind. Sind aber Kinder vorhanden, reduziert sich bei den Frauen die durchschnittliche Anzahl Arbeitsstunden um die Hälfte (Bühler und Heye 2005).

Inwiefern angeglichene Bildungschancen und erhöhte und konstantere Arbeitsmarktintegration auch in verbesserte Einkommens- und Karrierechancen umgemünzt werden, ist dagegen nicht vollständig geklärt. Mehrere Studien belegen, dass sich die geschlechtsspezifischen Barrieren in der Bildung zwar aufgelöst haben, im Berufsleben aber fortbestehen (vgl. den Forschungsstand in Buchmann et al. 2002 sowie Hecken 2006). Engelage und Hadjar (2008) untersuchen in diesem Zusammenhang für die Schweiz die Frage, ob sich eine Promotion beim Berufseinstieg für Frauen gleichermaßen auszahlt wie für Männer. Sie können erstens nachweisen, dass promovierte Frauen in allen Fächern weniger verdienen als Männer, insbesondere in den Ingenieurs- und Sozialwissenschaften. Zweitens zeigen sich deutliche Geschlechtereffekte zuungunsten der Frauen, wenn der Lohnvorteil einer Promotion im Vergleich zu einem Masterabschluss betrachtet wird. Frauen haben durch eine Promotion, Ausnahme sind die Ingenieurwissenschaften, geringere Lohnvorteile als Männer. Die Promotion lohnt sich offenbar mehr für die Männer. Ebenfalls promovierte Frauen und Männer in der Schweiz haben Engelage und Schubert (2009) hinsichtlich der Frage der Ausbildungsadäquatheit der Beschäftigung fünf bis zehn Jahre nach dem Doktorat untersucht. Dabei zeigt sich, dass der Zugang zu Führungspositionen für Frauen wesentlich schwieriger ist als für Männer, was bedeutet, dass sie hinsichtlich vertikaler ausbildungsadäquater Beschäftigung benachteiligt sind. Diese inadäquate Beschäftigung kann vor allem auf die überwiegend von Frauen ausgeübte Teilzeitarbeit zurückgeführt werden. 
Hecken (2006) untersucht in einer Kohortenstudie in der Schweiz den Zusammenhang von gestiegener Bildungsbeteiligung und höheren Bildungsniveaus mit dem erreichten Berufsstatus. Auch sie kommt zum Schluss, dass Frauen ihre (verbesserten) Ausbildungsabschlüsse - auch unter Kontrolle der Kohorten - nicht gleich gut in beruflichen Status umwandeln können wie Männer. Für jedes weitere Schuljahr ist die Bildungsrendite der Frauen geringer als jene der Männer. Heirat und insbesondere Teilzeitarbeit verringern diese Renditen nochmals. Hecken begründet dies einerseits mit der in der Schweiz nach wie vor ausgeprägten geschlechtsspezifischen Segregation der Ausbildungen und ausgeübten Berufe, andererseits mit der Tatsache, dass der zunehmenden Integration von Frauen ins Erwerbsleben nicht im gleichen Maße Veränderungen in den Mustern der familiären Arbeitsteilung gefolgt sind. Den Geschlechterordnungen in Bildung, Beruf und Familie kommen, so können wir festhalten, eine entscheidende Rolle zu für die Frage der Umsetzung verbesserter Bildungschancen von Frauen in berufliche Integrations- und Laufbahnchancen.

\section{Geschlechterverhältnisse im Lebensverlauf}

Mayer und Blossfeld (1990) haben schon vor zwanzig Jahren in dem für die Analyse der gesellschaftlichen Konstruktion von Ungleichheit im Lebensverlauf richtungsweisenden Aufsatz anhand empirischer Befunde aufgezeigt, dass die Berücksichtigung von lebenszeitlichen Aspekten der Ungleichheit und die Verknüpfung individueller Lebensläufe mit der Ebene gesellschaftlicher Institutionen für eine Beurteilung der Unterschiede von Frauen und Männern unverzichtbar ist, und dass die vergangene Lebensgeschichte die zukünftigen Lebenschancen endogen mitbestimmt.

Krüger und Levy $(2000,2001)$ haben diese lebenslauftheoretische Perspektive ergänzt und erweitert und mit Blick auf die "verlinkten Leben" von Paaren und Familien dafür plädiert, nicht nur die Laufbahnen von Individuen in den Blick zu nehmen, um die komplexen Konstruktionsprozesse von geschlechtsspezifischen Ungleichheiten über den Lebenslauf hinweg zu verstehen. Ihr institutionenorientierter Lebenslaufansatz fokussiert neben normativkulturellen im Weiteren strukturelle, in und durch Organisationen geformte Handlungsspielräume oder -imperative, welche die Lebensläufe von Männern und Frauen strukturieren. Insbesondere verweisen sie auf die folgenden Dimensionen:

- Neben der Konzeption des Lebenslaufes als Sequenz von drei relevanten LebenslaufInstitutionen (Bildung, Arbeitsmarkt, Pension), welche in einer diachronen Abfolge durchlebt werden, wird in einer synchronen Perspektive die gleichzeitige Einbindung in 
verschiedene soziale Institutionen, insbesondere auch in die Paarbeziehung und Familie als bedeutsam bewertet. Die Institution Familie führt verschiedene Lebensläufe und Lebenspläne zusammen: Partner mit ihren unterschiedlichen beruflichen Aspirationen und Einbindungen, Kinder mit ihren Beziehungen und (Bildungs-)Aktivitäten, weitere Familienmitgliedern (z.B. Großeltern) mit ihren Bedürfnissen (Pflege) oder Unterstützungen (Kinderbetreuung). Dies erfordert, Lebensverläufe nicht als eine Angelegenheit von Einzelpersonen, sondern als Paar- oder Familienkonstruktionen zu verstehen.

- Neben der Institution Familie ist die Strukturierungskraft von Anliegerinstitutionen mitzubedenken. Moderne Gesellschaften sind funktional stark ausdifferenziert in verschiedene Subsysteme und Organisationen, welche das Alltagsleben und die Lebensläufe von Individuen und Familien prägen. Zu denken ist u.a. an Anforderungen und kulturelle Normen im Beschäftigungssystem (z.B. Flexibilität) oder Angebote, Öffnungs- und Betreuungszeiten wichtiger privater wie staatlicher Versorgungsinstitutionen (Schule, familienergänzende Betreuungsinstitutionen) (vgl. auch Buchmann und Charles 1995). Diese Institutionen erleichtern oder erschweren die Vereinbarung von Kindern und Erwerbsarbeit und damit die innerfamiliären Muster von Arbeitsteilung.

- Geschlecht als Masterstatus strukturiert die Lebensläufe ungleich für Männer und Frauen. Auf der Mikroebene wirkt Geschlecht durch ein "doing gender" in den alltäglichen Interaktionen der Akteure in einer durch Zweigeschlechtlichkeit strukturierten Welt. Auf der Meso-Ebene ist Geschlecht als Strukturkategorie in mannigfaltiger Form in die Institutionen insbesondere des Bildungs-, Beschäftigungs- und Familiensystems und die Verknüpfungen und Interdependenzen der drei Systeme eingelassen. ${ }^{10}$

Die Bedeutung von geschlechtsspezifischen Bildungsungleichheiten für Berufsleben und Karriere, so unsere These, kann nur unter Bezugnahme auf diese komplexe und umfassende Perspektive theoretisch wie empirisch angemessen bearbeitet und beantwortet werden. Dies soll zum Abschluss anhand einiger ausgewählter Resultate einer Längsschnittuntersuchung zu geschlechtsspezifischen Laufbahnchancen in der Wissenschaft illustriert werden. In der vom Bundesamt für Statistik betreuten Panel-Studie wurden alle im Jahre 2002 in der Schweiz

10 In Studien zu Erwerbsverläufen von Frauen werden die (trotz erfolgreicher Bildungsabschlüsse) eingeschlagenen statusgeringeren Wege nicht selten damit erklärt, dass Frauen - ihre zukünftige Rolle als Mutter und Ehefrau antizipierend - entsprechende Laufbahnentscheidungen treffen, als würden die sozialen Verhältnisse in der Institution Familie nicht mit der Arbeitssphäre zusammenhängen. 
Doktorierten ein Jahr (2003) und fünf Jahre (2007) nach Abschluss der Dissertation zu ihrer weiteren beruflichen Entwicklung befragt. Zusätzlich wurden mit einer ausgewählten Stichprobe vertiefende Interviews geführt (vgl. Leemann und Stutz 2008). Dank Zusatzmodulen in den beiden Panels zu Bedingungen und Entwicklungen von wissenschaftlichen Laufbahnen konnte die Karriereentwicklung von Nachwuchsforschenden analysiert werden, wobei die Ursachen und Ausprägungen der "Leaky Pipeline", des überproportionalen Verlusts weiblichen wissenschaftlichen Nachwuchses, im Zentrum stand.

Bei gleichem Bildungsniveau der Befragten (Promotion) zeigen sich innerhalb des Beobachtungszeitraums von fünf Jahren für eine erfolgreiche wissenschaftliche Laufbahn ungleiche Entwicklungen nach Geschlecht, welche auch im weiteren Berufsverlauf geringere Karrierechancen von Frauen erwarten lassen. Geschlecht als Masterstatus macht sich bei der Förderung und Integration der Nachwuchsforschenden bemerkbar. So haben

Nachwuchswissenschaftlerinnen eine signifikant geringere Chance - weniger als halb so groß wie männliche Nachwuchskräfte - in der Postdocphase einen Professor oder eine Professorin zu finden, der/die sie im Sinne eines Mentorings entscheidend unterstützt und fördert (Leemann et al. 2010a). Auch die Einbindung von Frauen in internationale Netzwerke ist zum Befragungszeitpunkt 2007 signifikant schlechter als jene ihrer männlichen Kollegen (Leemann et al. 2010b). Dies kann mitunter die Tatsache erklären, dass die weiblichen Nachwuchswissenschaftler seit dem Doktorat im Durchschnitt nur knapp zwei Drittel so viel publiziert haben wie die männlichen (Leemann und Stutz 2008).

Auch die Familiensituation der Nachwuchskräfte ist durch geschlechtsspezifische Strukturen gekennzeichnet (vgl. im Folgenden auch Leemann et al. 2010a). Zum Befragungszeitpunkt 2007 leben die Akademikerinnen und Akademiker zwar gleich häufig in einer Partnerschaft. Die Gründung einer Familie ist für Frauen im Vergleich zu den Männern jedoch schwieriger mit einer weiteren Karriere zu verbinden. Dies zeigt sich anhand dreier Phänomene. Erstens haben die Frauen fünf Jahre nach dem Doktorat weniger häufig Kinder. Im Hochschulbereich tätige Frauen haben zu 32 Prozent mindestens ein Kind, wenn sie außerhalb der Hochschule arbeiten, ist dieser Anteil mit 38 Prozent etwas höher. Männer im Hochschulbereich haben in 43 Prozent der Fälle mindestens ein Kind, wenn sie in andere Tätigkeitsbereiche gewechselt haben, sind sie in 57 Prozent der Fälle Vater geworden. Wie weitere Auswertungen zeigen, planen Frauen, falls sie (noch) keine Kinder haben, auch weniger Kinder für die Zukunft als Männer. Die Geschlechterunterschiede bezüglich Elternschaft werden sich demnach noch vergrößern. Diese Ergebnisse verweisen darauf, dass in der Schweiz die Vereinbarung einer wissenschaftlichen Laufbahn mit der Gründung einer 
Familie nicht nur durch die Geschlechtszugehörigkeit, sondern auch durch institutionelle Faktoren des wissenschaftlichen Feldes erschwert wird, bei beiden Geschlechtern.

Zweitens verändert sich das zuvor noch relativ gleichartige erwerbsbezogene Muster der Arbeitsteilung der Paare, wenn die Paartypen befragte Akademikerin \& Partner(in) mit befragten Akademiker \& Partner(in) ${ }^{11}$ betrachtet werden, eklatant, wenn Kinder dazu stoßen. Weibliche Doktorierte mit Kindern sind zwar größtenteils (85 Prozent) erwerbstätig, jedoch häufig nur in Teilzeit (66 Prozent der Befragten). In ca. 30 Prozent der Fälle sind auch ihre Partner Teilzeit erwerbstätig, die restlichen 70 Prozent arbeiten Vollzeit. Haben dagegen männliche Doktorierte Kinder, ändern sie ihr Erwerbsverhalten nicht und sind mit wenigen Ausnahmen weiterhin berufstätig, zum größten Teil Vollzeit (80 Prozent). Ihre Partnerinnen aber reduzieren häufig auf Teilzeit (50 Prozent) oder unterbrechen die Erwerbstätigkeit ganz (40 Prozent).

Drittens zeigt die Aufteilung der Verantwortung für die Kinderbetreuung in Paarhaushalten auch in dieser Gruppe von Hochqualifizierten die bekannten Muster innerfamiliärer Arbeitsteilung. Die Hälfte der Väter kann sich auf eine Partnerin abstützen, welche die Kinderbetreuung an Werktagen vollumfänglich übernimmt oder organisiert. Dies ist bei den befragten Müttern kaum der Fall. Sie sind immer in die Betreuung involviert, indem sie selbst Betreuungsaufgaben übernehmen und die Betreuung mit Hilfe von Drittpersonen und Betreuungsinstitutionen organisieren. ${ }^{12}$

Die Relevanz der "linked lives" - oder in diesem Kontext der "dual career couples" macht sich ganz konkret bemerkbar bei der für eine wissenschaftliche Laufbahn bedeutsamen geografischen akademischen Mobilität. Unsere Untersuchungen zeigen, dass Wissenschaftlerinnen und Wissenschaftler, welche zum Zeitpunkt des Doktorats in einer Paarbeziehung lebten, sich in der Postdocphase signifikant seltener für eine wissenschaftliche Phase ins Ausland begeben. Der im wissenschaftlichen Feld geforderte Idealtypus eines Wissenschaftlerunternehmers, "nomadic and monadic, de-territorialised, disembodied and disembedded" (Leemann 2010) ist männlich, jung, stammt aus akademischer Herkunftsfamilie, hat (noch) keine Kinder, lebt nicht in fester Partnerschaft und wird wissenschaftlich durch einen Mentor und Institutionen der Forschungsförderung unterstützt.

\footnotetext{
${ }^{11}$ Da es sich in den meisten Fällen um heterosexuelle Paarkonstellationen handeln wird, ist im Folgenden das Geschlecht der Partner jeweils entsprechend als männlich oder weiblich angenommen.

${ }^{12}$ In der Schweiz ist in der Gesamtbevölkerung die traditionelle Versorgerehe (der Mann arbeitet Vollzeit, die Frau Teilzeit oder ist nicht erwerbstätig und übernimmt den größten Teil der Familienarbeit) nach wie vor vorherrschend (Strub et al. 2005; Baumgartner 2006). Frauen und Männer sind in der Folge sehr ungleich in die Sphären von Familien- und Erwerbsleben eingebunden, sobald Kinder vorhanden sind.
} 
Wie in Kapitel 4 und 5 gezeigt, haben hochqualifizierte Frauen (mit Promotion) bei der Verfolgung einer Karriere in- und außerhalb der Wissenschaft größere Hindernisse zu überwinden als hochqualifizierte Männer. Sie verdienen weniger, werden weniger gefördert und sind schon wenige Jahre nach der Promotion in Führungspositionen untervertreten.

\section{Zum Zusammenhang von Bildung, Beruf und Karriere aus Geschlechterperspektive:}

\section{Ein Fazit}

Wie ist nun das Aufholen der Frauen - oder gar Überholen der Männer - im Bildungsbereich im Hinblick auf die Frage von Persistenz und Wandel von gesellschaftlichen Geschlechterungleichheiten abschließend einzuordnen? Ziel des Aufsatzes war es, eine Interpretationsfolie zu dieser Frage zu skizzieren und eine Einschätzung vorzunehmen. Zusammenfassend können wir festhalten, dass Untersuchungen von Geschlechterungleichheiten im Bildungssystem, welche auf der Ebene der ISCEDKlassifikationen operieren, zu kurz greifen. Sie können die in den Kategorien zusammengefassten unterschiedlichen Bildungswege und Bildungstitel und die darin angelegten ungleichen Beschäftigungschancen nicht erfassen. Zwei Charakteristiken des schweizerischen wie auch des deutschen Bildungssystems erfordern differenziertere Analysen. Zum einen ist dies die ausgeprägte Binarität des Systems, mit den biografisch früh eingeleiteten und voneinander getrennten beruflichen und allgemeinbildenden Pfaden, welche in sich nach Fachrichtungen weiter stark ausdifferenziert sind. Zum anderen ist dies der sich nach wie vor stark auf die Institution der Beruflichkeit und der Zertifizierung stützende Arbeitsmarkt, der eine enge Verbindung zum Bildungssystem schafft (Buchmann und Sacchi 1998).

Wir haben den Blick auf den Zusammenhang zwischen Bildung, Beruf und Karriere gerichtet und herausgearbeitet, dass Analysen von Transformationen oder Beharrungstendenzen des Geschlechterverhältnisses Phänomene und Wandlungsprozesse in den Institutionen Bildung, Beschäftigung, Familie und Lebenslauf untersuchen und aufeinander beziehen müssen (vgl. Kraus 2006). Empirische Ergebnisse für die gegenwärtige Situation in der Schweiz verweisen auf anhaltende sozial relevante Geschlechterordnungen im Dreieck Bildung, Beruf und Familie, welche im Ergebnis dafür verantwortlich sind, dass Frauen ihre Bildungsgewinne der letzten dreißig Jahre nicht angemessen in berufliche Chancen umwandeln konnten. Es sind dies erstens die in den Ausbildungswegen angelegten 
Geschlechtersegregationen, welche Frauen und Männer in unterschiedliche berufliche Felder und zu ungleichen beruflichen Beschäftigungschancen führen, zweitens Benachteiligungen von Frauen bei der Umwandlung von gleichen oder vergleichbaren Bildungstiteln in berufliche Positionen und Laufbahnchancen, sowie drittens der Umstand, dass Frauen ihr Bildungskapital durch die ihnen gesellschaftlich zugewiesene Reproduktionsarbeit nicht zu den gleichen Bedingungen in Einkommen, Berufsstatus und formale Autorität ummünzen können und deshalb im Bereich der Familie (mit dem Verzicht auf Kinder) oder im Beruf (mit Teilzeitarbeit und Karriereverzicht) zurückstecken müssen. Befürchtungen, dass sich das Geschlechterverhältnis in den nächsten Jahren nun zu Ungunsten der Männer umkehren wird, scheinen angesichts der großen Persistenz der Geschlechterungleichheiten in Beschäftigung und Familie zurzeit unbegründet. 


\section{Literatur}

Abraham, Martin und Jürg Arpagaus. 2008. Wettbewerb, soziales Umfeld oder gezielte Lebensplanung? Determinanten der horizontalen Geschlechtersegregation auf dem Lehrstellenmarkt. Soziale Welt 59: 205-225.

Babel, Jacques and Pascal Strubi. 2010. Bildungsperspektiven Szenarien 2010 -2019 für die Hochschulen. Neuchâtel: Bundesamt für Statistik.

Baumgartner, A. Doris. 2006. Familienarbeit, Erwerbsmuster und Arbeitsteilung im Haushalt, Hrsg. Informationen aus der Demografie 4/2005. Neuchâtel: Bundesamt für Statistik.

BBT, Bundesamt für Berufsbildung und Technologie. 2010. Berufsbildung in der Schweiz. Fakten und Zahlen. Bern: BBT.

Becker, Rolf und Andreas Hadjar. 2009. Meritokratie: Zur gesellschaftlichen Legitimation ungleicher Bildungs-, Erwerbs- und Einkommenschancen in modernen Gesellschaften. In Lehrbuch der Bildungssoziologie, Hrsg. Rolf Becker, 35-59. Wiesbaden: VS Verlag für Sozialwissenschaften.

BfS, Bundesamt für Statistik. 2010. Statistik der beruflichen Grundbildung 2009. Neuchâtel: Bundesamt für Statistik

Bieri Buschor, Christine, Stefan Denzler und Andrea Keck. 2008. Welche Maturandinnen und Maturanden wählen welche Studienfächer? Netzwerk. Die Zeitschrift der Wirtschaftsbildung Schweiz 102: 34-43.

Blossfeld, Hans-Peter. 1989. Kohortendifferenzierung und Karriereprozess: eine Längsschnittstudie über die Veränderung der Bildungs- und Berufsschancen im Lebenslauf. Frankfurt a.M.: Campus.

Borkowsky, Anna. 2000. Frauen und Männer in der Berufsbildung der Schweiz. Schweizerische Zeitschrift für Bildungswissenschaften 22: 279-294.

Bornschier, Volker und Doris Aebi. 1992. Rolle und Expansion der Bildung in der modernen Gesellschaft - Von der Pflichtschule bis zur Weiterbildung. Schweizerische Zeitschrift für Soziologie 18: 539-567.

Bridges, William P. 2003. Rethinking gender segregation and gender inequality: Measures and meanings. Demography 40: 543-568.

Buchmann, Marlis und Maria Charles. 1995. Organizational and institutional factors in the process of gender stratification: Comparing social arrangements in six European countries. International Journal of Sociology 25: 66-95.

Buchmann, Marlis und Irene Kriesi. 2008. Feminisierung der Arbeitswelt. In Tagesstrukturen als sozial-und bildungspolitische Herausforderung: Erfahrungen und Kontexte, Hrsg. Sabina Larcher Klee und Bettina Grubenmann, 51-64. Bern: Haupt.

Buchmann, Marlis und Irene Kriesi. 2009. Escaping the gender trap: Young women's transition into nontraditioinal occupations. In Transition from School to Work. Globalisation, Individualisation, and Patterns of Diversity, Hrsg. Ingrid Schoon und Rainer K. Silbereisen, 193-215. Cambridge/New York: Cambridge University Press.

Buchmann, Marlis, Irene Kriesi, Andrea Pfeifer und Stefan Sacchi. 2002. halb drinnen - halb draussen. Analysen zur Arbeitsmarktintegration von Frauen in der Schweiz. Chur, Zürich: Rüegger.

Buchmann, Marlis und Stefan Sacchi. 1998. The transition from school to work in Switzerland: Do characteristics of the educational system and class barriers matter? In From school to work. A comparative study of educational qualifications and occupational destinations, Hrsg. Yossi Shavit und Walter Müller, 407-442. Oxford: Clarendon Press.

Bühler, Caroline und Corinna Heye. 2005. Fortschritte und Stagnation in der Gleichstellung der Geschlechter 1970-2000. Neuchâtel: Bundesamt für Statistik.

Charles, Maria. 2005. Entwicklung der beruflichen Segregation nach Geschlecht und nach Staatsangehörigkeit in der Schweiz, 1970-2000. Neuchâtel: Bundesamt für Statistik.

Charles, Maria und Karen Bradley. 2009. Indulging our gendered selves? Sex segregation by field of study in 44 countries. American Journal of Sociology 114: 1475-1521.

Charles, Maria und Marlis Buchmann. 1994. Assessing Micro-Level Explanations of Occupational Sex Segregation: Human Capital Development and Labor Market Opportunities in Switzerland. Schweizerische Zeitschrift für Soziologie 20: 595-620.

Cortina, Kai S., Jürgen Baumert, Achim Leschinsky, Karl Ulrich Mayer und Luitgard Trommer. 2008. Das Bildungswesen in der Bundesrepublik Deutschland. Strukturen und Entwicklungen im Überblick. Reinbek: Rowohlt.

Criblez, Lucien. 2001. Bildungsexpansion durch Systemdifferenzierung - am Beispiel der Sekundarstufe II in den 1960er- und 1970erJahren. Schweizerische Zeitschrift für Bildungswissenschaften 23: 95-116.

Denzler, Stefan und Stefan C. Wolter. 2008. Selbstselektion bei der Wahl eines Lehramtsstudiums: Zum Zusammenspiel individueller und institutioneller Faktoren. Beiträge zur Hochschulforschung 30: 112141.

Dubach, Philipp. 2010. Leaky Pipeline: der überproportionale Verlust von Frauen in wissenschaftlichen Laufbahnen in der Schweiz. In Forschungsförderung aus Geschlechterperspektive. Zugang, Bedeutung und Wirkung in wissenschaftlichen Laufbahnen, Hrsg. Regula Julia Leemann und Heidi Stutz, 39-61. 
Zürich: Rüegger.

Eidgenössische Kommission für Frauenfragen. 2010. Mädchenbildung und Koedukation. In Frauen Macht Geschichte. Zur Geschichte der Gleichstellung in der Schweiz 1848 - 2000, Hrsg. Bundesamt für Statistik. http://www.ekf.admin.ch/themen/00504/00558/00559/00560/ (Stand: 2. Oktober 2010).

Engelage, Sonja und Andreas Hadjar. 2008. Promotion und Karriere - Lohnt es sich zu promovieren? Eine Analyse der Schweizerischen Absolventenstudie. Schweizerische Zeitschrift für Soziologie 34:71-93.

Engelage, Sonja und Frank Schubert. 2009. Promotion und Karriere - Wie adäquat sind promovierte Akademikerinnen und Akademiker in der Schweiz beschäftigt? Zeitschrift für Arbeitsmarktforschung 41:213-233.

Flückiger, Yves und Jean-Marc Falter. 2004. Bildung und Arbeit. Entwicklung des Arbeitsmarktes in der Schweiz. Neuchâtel: Bundesamt für Statistik.

Franzen, Axel, Anna Hecken und Christoph Kopp. 2004. Bildungsexpansion und die geschlechtsspezifische Segregation an Schweizer Hochschulen. Soziale Welt 55:317-336.

Gonon, Philipp. 1998. Berufliche Bildung zwischen Zunft, Handelsfreiheit und Demokratie. Bildungsforschung und Bildungspraxis 20: 419-431.

Gonon, Philipp. 2001. Young women in swiss vocational education and in business matura (school leaving examination). In Gender perspectives on vocational education. Historical, cultural and policy aspects, Hrsg. Philipp Gonon, Kurt Häfeli, Anja Heikkinen und Iris Ludwig, 57-72. Bern: Peter Lang.

Graf, Martin und Markus Lamprecht. 1991. Der Beitrag des Bildungssystems zur Konstruktion sozialer Ungleichheit. In Das Ende der sozialen Schichtung? Zürcher Arbeiten zur gesellschaftlichen Konstruktion von sozialer Lage und Bewusstsein in der westlichen Zentrumsgesellschaft, Hrsg. Volker Bornschier, 73-96. Zürich: Seismo.

Hecken, Anna Etta. 2006. Bildungsexpansion und Frauenerwerbstätigkeit. In Die Bildungsexpansion. Erwartete und unerwartete Folgen, Hrsg. Andreas Hadjar und Rolf Becker, 122-155. Wiesbaden: VS Verlag für Sozialwissenschaften.

Hirschi, Andreas. 2009. Eine typologische Analyse des schweizerischen Lehrstellenmarktes: Strukturelle Benachteiligung von jungen Frauen. Schweizerische Zeitschrift für Bildungswissenschaften 31: $317-$ 333.

Holst, Elke und Anne Busch. 2009. Der „Gender Pay Gap“ in Führungspositionen der Privatwirtschaft in Deutschland. SOEPpapers 169.

Hupka, Sandra. 2003. Ausbildungssituation und -verläufe: Übersicht. In Wege in die nachobligatorische Ausbildung. Die ersten zwei Jahre nach Austritt aus der obligatorischen Schule. Zwischenergebnisse des Jugendlängsschnitts TREE, Hrsg. Jaques Amos, Edi Böni, Mario Donati, Sandra Hupka, Thomas Meyer und Barbara E. Stalder, 33-58. Neuchâtel: Bundesamt für Statistik.

Imdorf, Christian. 2005. Schulqualifikation und Berufsfindung. Wie Geschlecht und nationale Herkunft den Übergang in die Berufsbildung strukturieren. Wiesbaden: VS Verlag für Sozialwissenschaften.

Jann, Ben. 2008. Erwerbsarbeit, Einkommen und Geschlecht. Studien zum Schweizer Arbeitsmarkt. Wiesbaden: VS Verlag für Sozialwissenschaften.

Jenzer, Carlo. 1998. Schulstrukturen als historisch gewachsenes Produkt bildungspolitischer Vorstellungen. Blitzlichter in die Entstehung der schweizerischen Schulstrukturen. Bern: Peter Lang.

Jurczyk, Karin. 1993. Flexibilisierung für wen? Zum Zusammenhang von Arbeitszeiten und Geschlechterverhältnissen. In Die Arbeit des Alltags. Beiträge zu einer Soziologie der alltäglichen Lebensführung, Hrsg. Jurczyk, Karin und Maria S. Rerrich, 346-374. Freiburg/Br.: Lambertus.

Krais, Beate. 1983. Bildung als Kapital: Neue Perspektiven für die Analyse der Sozialstruktur? In Soziale Ungleichheiten. Sonderband 2 der Sozialen Welt, Hrsg. Reinhard Kreckel, 199-220. Göttingen: Schwartz.

Kraus, Katrin. 2006. Better educated, but not equal: women between general education, VET, the labour market and the family in Germany. Journal of Vocational Education and Training 58: 409-422.

Krüger, Helga. 1995. Dominanzen im Geschlechterverhältnis: Zur Institutionalisierung von Lebensläufen. In Das Geschlechterverhältnis als Gegenstand der Sozialwissenschaften, Hrsg. Regina Becker-Schmidt und Gudrun-A. Knapp, 195-219. Frankfurt; New York: Campus.

Krüger, Helga und René Levy. 2000. Masterstatus, Familie und Geschlecht. Vergessene Verknüpfungslogiken zwischen Institutionen des Lebenslaufs. Berliner Journal für Soziologie 10: 379-401.

Krüger, Helga und René Levy. 2001. Linking Life courses, Work, and the Family: Theorizing a not so Visible Nexus between Woman and Men. Canadian Journal of Sociology 26: 145-166.

Kunz, Barbara. 2010. Studierende an den universitären Hochschulen 2009/10. Neuchâtel: Bundesamt für Statistik.

Kupfer, Antonia. 2010. The socio-political significance of changes to the vocational education system in Germany. British Journal of Sociology of Education 31: 85-97.

Leemann, Regula Julia. 2010. Gender inequalities in transnational academic mobility and the ideal type of an academic entrepreneur. Discourse, Studies in the Cultural Politics of Education 31: 609-625.

Leemann, Regula Julia, Stefan Boes und Sandra Da Rin. 2010a. La perte plus que proportionnelle des femmes 
dans les parcours académiques. Quelques résultats quant aux caractéristiques sociales du champ scientifique et à ses genrés d'exclusion. In Le plafond de fer de l'université. Femmes et carrières, Hrsg. Farinaz Fassa et Sabine Kradolfer, S. 127-153. Zürich: Seismo.

Leemann, Regula Julia, Philipp Dubach und Stefan Boes. 2010b. The leaky pipeline in the Swiss university system: Identifying gender barriers in postgraduate education and networks using longitudinal data. Swiss Journal of Sociology 36: 299-323.

Leemann, Regula Julia und Andrea Keck. 2005. Der Übergang von der Ausbildung in den Beruf. Die Bedeutung von Qualifikation, Generation und Geschlecht. Neuchâtel: Bundesamt für Statistik.

Leemann, Regula Julia und Heidi Stutz. 2008. Geschlecht und Forschungsförderung (GEFO). Bern, Schweizerischer Nationalfonds. http://www.snf.ch/D/ueberuns/foerderungspolitik/Seiten/Gleichstellung.aspx (Stand: 2. Oktober 2010).

Mayer, Karl-Ulrich und Hans-Peter Blossfeld. 1990. Die gesellschaftliche Konstruktion sozialer Ungleichheit im Lebensverlauf. In Lebenslagen, Lebensläufe, Lebensstile. Sonderband 7 der Sozialen Welt, Hrsg. Peter A. Berger und Stefan Hradil, 297-318. Göttingen: Otto Schwartz Verlag.

Meyer, Thomas. 2009. Can "vocationalisation" of education go too far? The case of Switzerland. European Journal of Vocational Training 46: 28-40.

OECD. 2008. Education at a glance. OECD indicators. Paris: OECD.

OECD. 2009. Education at a glance. OECD indicators. Paris: OECD.

Ramseier, Erich, Jürgen Allraum, Ursula Stalder, François Grin, Roberta Alliata, Stephan Müller, Ivo Willimann, Edo Dozio, Peter Labudde, Katharina Maag Merki, Elisabetta Pagnossin und Eugen Stocker. 2005. Evaluation der Maturitätsreform 1995 (EVAMAR). Bern: Staatssekretariat für Bildung und Forschung.

Rieger, Andreas. 2001. Bildungsexpansion und ungleiche Bildungspartizipation am Beispiel der Mittelschulen im Kanton Zürich, 1830 bis 1980. Schweizerische Zeitschrift für Bildungswissenschaften 23: 41-72.

Sacchi, Stefan. In Vorbereitung. Arbeitsmarktstrukturen, Löhne und Beschäftigungschancen. Zürich: Seismo.

Schweizerische Koordinationsstelle für Bildungsforschung SKBF. 2010. Bildungsbericht Schweiz, 2010. Aarau: SKBF.

Seibert, Holger, Sandra Hupka-Brunner und Christian Imdorf. 2009. Wie Ausbildungssysteme Chancen verteilen. Berufsbildungschancen und ethnische Herkunft in Deutschland und der Schweiz unter Berücksichtigung des regionalen Verhältnisses von betrieblichen und schulischen Ausbildungen. Kölner Zeitschrift für Soziologie und Sozialpsychologie 61: 595-620.

Smith, Emer und Stephanie Steinmetz. 2008. Field of study and gender segregation in European labour markets. International Journal of Comparative Sociology 49: 257-281.

Solga, Heike. 2005. Meritokratie - die moderne Legitimation ungleicher Bildungschancen. In Institutionalisierte Ungleichheiten. Wie das Bildungswesen Chancen blockiert, Hrsg. Peter A. Berger and Heike Kahlert, 19-38. Weinheim, München: Juventa Verlag.

Solga, Heike and Dirk Konietzka. 2000. Das Berufsprinzip des deutschen Arbeitsmarktes. Ein geschlechtsneutraler Allokationsmechanismus? Schweizerische Zeitschrift für Soziologie 26: 111-147.

Strub, Silvia, Eveline Hüttner und Jürg Guggisberg. 2005. Arbeitsteilung in Paarhaushalten. Aufteilung von bezahlter und unbezahlter Arbeit in der Schweiz. Neuchâtel: Bundesamt für Statistik.

Trappe, Heike. 2006. Berufliche Segregation im Kontext. Über einige Folgen geschlechtstypischer Berufsentscheidungen in Ost- und Westdeutschland. Kölner Zeitschrift für Soziologie und Sozialpsychologie 58: 50-78. 